\title{
Rôle des lipides dans la régulation du comportement alimentaire
}

\author{
Dany GAILLARD \\ Céline MARTIN \\ Patricia PASSILLY-DEGRACE \\ Philippe BESNARD
}

Physiologie de la nutrition, UMR Inserm U 866, Ecole nationale supérieure de biologie appliquée à la nutrition et à I'alimentation (ENSBANA), Université de Bourgogne,

1 Esplanade Erasme, 21000 Dijon, France

<pbesnard@u-bourgogne.fr>

Physiologiquement, le comportement alimentaire résulte de l'intégration de signaux multiples (nerveux, hormonaux, métaboliques) convergeant vers des zones spécifiques du système nerveux central pour y être intégrés. Ces informations d'origine nutritionnelle conditionnent à la fois le choix des aliments à consommer (préférence ou aversion) et les déterminants de la prise alimentaire : initiation (faim), durée (rassasiement) et intervalle entre les repas (satiété). Les régulations homéostatiques mises en jeu sont donc essentielles pour adapter les apports caloriques aux besoins énergétiques de l'organisme. En fait, la composition chimique des aliments est perçue par I'intermédiaire d'informations précoces d'origine somesthésique, olfactive et gustative déclenchées dès la mise en bouche de l'aliment et de signaux neuroendocrines et métaboliques plus tardifs d'origine post-ingestive puis post-absorptive. C'est notamment le cas des lipides alimentaires (figure 1) dont l'implication dans le contrôle du comportement alimentaire fait l'objet d'une recherche soutenue. En effet, on sait qu'une consommation lipidique exces-

AGL : acides gras libres

AGLC : acides gras à longue chaîne

Apo AIV : apolipoprotéine AIV

CCK : cholecystokinine

GLP-1 : glucagon-like protein 1

NTS : noyau du tractus solitaire

PYY : peptide YY

TG : triglycérides

TRC : taste receptor cells

\begin{abstract}
Obesity constitutes a major public health problem for the $21^{\text {st }}$ Century, with its epidemic spread worldwide, particularly in children. The overconsumption of fatty foods greatly contributes to this phenomenon. Rodents and humans display a spontaneous preference for lipid-rich foods. But why are lipids so attractive? What are the molecular mechanisms involved? The purpose of this mini review is to explore few basic questions raised by recent data providing evidences on the relationships between dietary lipids and feeding behavior.
\end{abstract}

Key words: lipid preference, feeding behaviour, addiction, health

sive et déséquilibrée (trop de graisses saturées et de cholestérol, rapport $\omega 6 / \omega 3$ trop élevé) joue un rôle non négligeable dans l'augmentation de la prévalence des pathologies de pléthore (obésité, diabète de type 2 , athérosclérose, hypertension, cancers). Pourquoi les lipides alimentaires sont-ils si attractifs? Comment régulent-ils la prise alimentaire ? Peut-on parler d'une addiction aux lipides? Ces questions soulevées par les travaux récents seront abordées dans cette mini-revue.

\section{La génétique influence-t-elle la préférence pour les lipides?}

La préférence pour les lipides est un phénomène commun à différentes espèces du règne animal. Ainsi, l'Homme et les rongeurs (rat, souris) présentent une préférence spontanée pour les lipides alimentaires [1-3]. D'anciennes données ont établi que les sujets obèses auraient une préférence accrue pour les aliments riches en graisses par rapport aux personnes minces $[4,5]$. Ces travaux suggèrent qu'une différence de sensibilité dans la perception des lipides alimentaires pourrait contribuer à l'apparition de ces troubles. On peut se demander quelle est la part de l'inné par rapport à l'acquis dans ce phénomène. Des travaux récents de notre laboratoire indiquent que des facteurs génétiques y jouent un rôle fondamental chez la souris.

Pendant longtemps on a considéré que seules la texture et l'olfaction étaient impliquées dans la préférence spontanée pour les lipides chez le mammifère. Des travaux récents réalisés chez le rongeur indiquent que le goût jouerait un rôle important dans ce comportement (voir les articles publiés dans $\mathrm{OCL}[6,7])$ ouvrant ainsi la perspective de l'existence d'un système de récepteur pour les graisses.

La perception des saveurs s'effectue par le biais de cellules réceptrices spécialisées (taste receptor cells, TRC) localisées dans les bourgeons du goût des papilles gustatives essentiellement trouvées dans l'épithélium lingual. Ces cellules expriment les systèmes de détection spécifiques à chacune des saveurs de base (sucré, salé, acide, amer, umami). Nous avons récemment montré que la protéine $\mathrm{CD} 36$, connue pour lier avec une très haute affinité les acides gras à longue chaîne (AGLC, nombre de carbones $\geq 16$ ) est abondamment exprimée au niveau de certaines TRC chez la souris [8]. Cette glycoprotéine transmembranaire s'est avérée être impliquée dans la perception des lipides. En effet, l'invalidation du gène codant pour le CD36 diminue de façon drastique la préférence spontanée pour les AGLC chez la souris, sans pour autant affecter sa perception du sucré ou de l'amer [8]. Les travaux récents de notre équipe ont permis de montrer que la perception orosensorielle des lipides alimentaires dépendante du CD36 lingual relève de la gustation $[9,10]$. Ces résultats inédits suggèrent donc qu'il existe une dimension génétique à la perception orosensorielle des lipides alimentaires chez le rongeur. Des travaux récents suggèrent qu'il existe aussi une perception gustative des lipides alimentaires chez l'Homme [11]. On ignore actuellement si le phénomène est CD36 dépendant. 


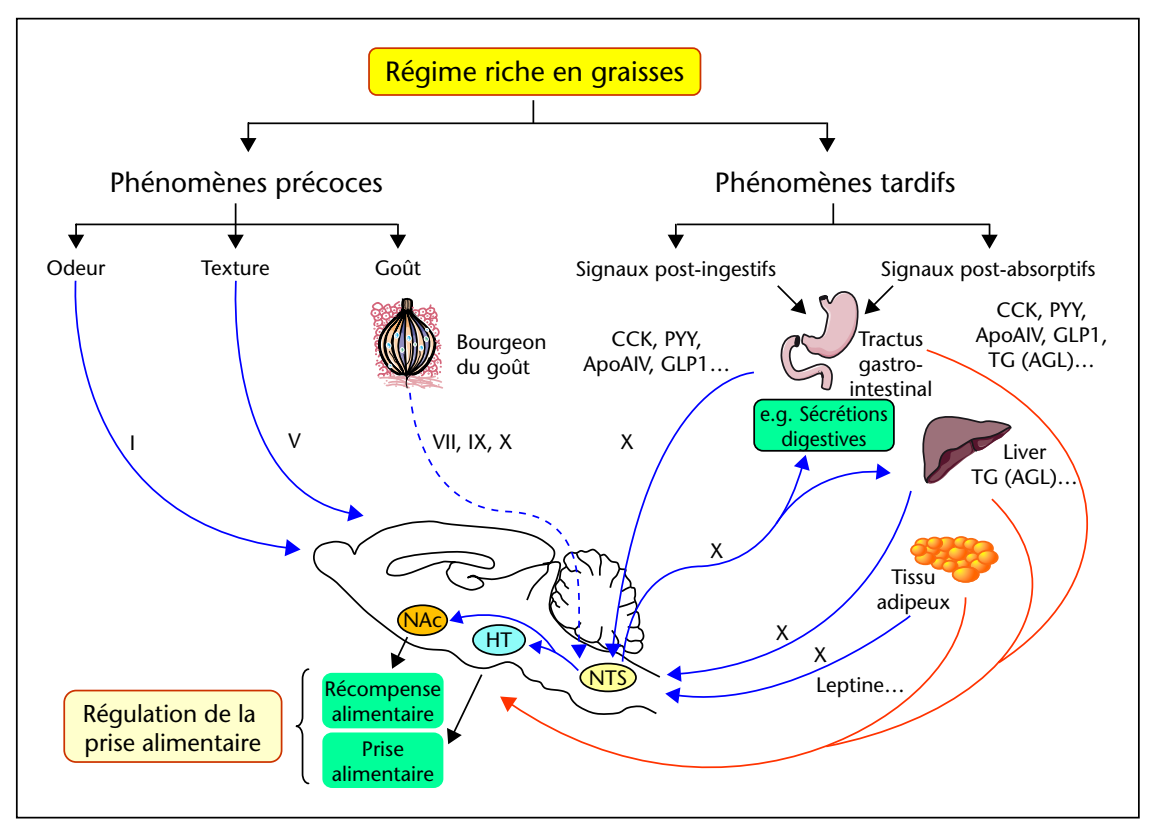

Figure 1. Représentation schématique du contrôle de la prise alimentaire par les lipides.

Les graisses alimentaires sont perçues par l'organisme via des phénomènes précoces impliquant l'odorat, le toucher et le goût et via des phénomènes plus tardifs d'origine post-ingestive et post-absorptive. Les signaux induits par ces modes de perception transitent par différentes voies nerveuses (en bleu) mais également par la circulation sanguine (en rouge). L'ensemble de ces signaux nerveux et endocrines (peptides, hormones, lipides) convergent au niveau du système nerveux central via le noyau du tractus solitaire (NTS) et rejoignent ainsi des zones impliquées dans le comportement alimentaire comme I'hypothalamus (HT) ou le noyau accumbens (NAc).I, nerf olfactif; $V$, nerf trijumeau; VII, chorde du tympan; IX, nerf glossopharyngien; X, nerf vague; AGL, acide gras libre; Apo AIV, apolipoprotéine AIV; CCK, cholecystokinine; GLP1, glucagon-like protein 1 ; PYY, peptide YY ; TG, triglycerides.

\section{Pourquoi les lipides sont-ils peu satiégogènes?}

Une fois ingérés, les lipides alimentaires déclenchent une cascade d'évènements visant à limiter la prise alimentaire. La cholécystokinine (CCK) joue un rôle essentiel dans cette régulation (figure 1). Cette hormone, produite par les cellules entéro-endocrines de type I essentiellement localisées au niveau du grêle proximal, est rapidement sécrétée suite à l'ingestion de lipides chez la plupart des mammifères dont I'Homme. Chez le rongeur, I'injection systémique de CCK s'accompagne d'un arrêt de la prise alimentaire. Cette régulation est la conséquence d'un contrôle paracrine d'afférences vagales intestinales exprimant le récepteur CCK1-R [12]. De ce fait, la vagotomie abolit l'effet satiétogène de la CCK. Cette régulation est cependant modulable puisque la consommation chronique d'un régime riche en lipides s'accompagne chez le rongeur d'une réduction de la réponse du nerf vague à l'injection de CCK [13]. Il a été récemment montré que cette désensibilisation est la conséquence d'une régulation dynamique du récepteur à CCK1-R dont le nombre au niveau des afférences vagales intestinales diminuerait rapidement consé- cutivement à une consommation de lipides [12]. Le nerf vague exprime aussi les récepteurs d'autres agents satiétogènes comme la glucagon-like protein-1 (GLP-1) et le peptide YY (PYY) qui sont sécrétés par l'iléon en réponse à une charge en lipides (figure 1). Comme pour le CCK1-R, ces récepteurs vagaux voient leur nombre diminué lors d'une consommation chronique de lipides [12]. Ce mécanisme limitant l'impact de ces hormones inhibitrices pourrait, en partie, expliquer pourquoi les lipides sont moins satiétogènes que les glucides ou les protéines.

Parallèlement à ces régulations neuroendocrines post-ingestives, des signaux satiétogènes sont produits consécutivement à l'absorption des lipides alimentaires. C'est le cas notamment de l'apolipoprotéine AIV (ApoAIV) qui est sécrétée par les entérocytes lors de la synthèse post-prandiale des chylomicrons (figure 1). Contrairement aux régulateurs post-ingestifs qui limitent la taille du repas (rassasiement), I'ApoAIV affecte l'intervalle entre deux repas consécutifs (satiété). De ce fait, l'injection systémique ou cérébroventriculaire d'ApoAIV diminue de façon dose-dépendante la prise alimentaire chez le rat [14]. Une fois encore, l'action satiégogène de l'Apo AIV décroît lors de régimes chroniquement riches en lipides. Le mécanisme à l'origine de cette désensibilisation n'est pas encore établi. II a été proposé que la leptine produite par le tissu adipeux (figure 1) soit en partie responsable de ce phénomène. En effet, l'expression de l'Apo AIV en réponse à une charge lipidique est plus faible chez des rats traités par la leptine [15]. La leptinémie étant particulièrement élevée au cours de l'obésité, une inhibition leptine-dépendante de la sécrétion de I'Apo AIV pourrait en partie expliquer la surconsommation alimentaire souvent observée chez les sujets et les animaux obèses [14].

\section{L'hypothalamus est-il une cible des lipides?}

Des expériences chez des souris dont les circulations sanguines ont été croisées (parabiose) ont permis de montrer que, parallèlement aux régulations nerveuses, des facteurs humoraux sont impliqués dans la régulation du comportement alimentaire [16]. On sait aujourd'hui que des agents satiétogènes dont la sécrétion est induite par les lipides (CCK, GLP-1, PYY336, leptine, Apo AIV) agissent directement au niveau du système nerveux central. Cette régulation est rendue possible en raison de la discontinuité de la barrière hémato-encéphalique notamment au niveau du noyau arqué hypothalamique (figure 2) dont le rôle dans le contrôle de la prise alimentaire est bien établi [17]. II a été récemment montré que les acides gras libres peuvent aussi moduler directement ce paramètre (figure 1). En effet, I'injection centrale d'acide oléique inhibe la prise alimentaire chez le rat [18]. Cet effet satiétogène semble être médié par des neurones spécifiques du noyau arqué sensibles aux acides gras [19]. Toutefois, les mécanismes moléculaires responsables de cette régulation neuronale ne sont pas encore établis. Une régulation directe par les acides gras de canaux ioniques, I'activation de récepteurs spécifiques (CD36 ?) ou encore la génération de métabolites comme le malonyl-CoA [18] sont des pistes plausibles. En bref, le transfert de petites quantités d'acides gras depuis la périphérie vers l'hypothalamus est interprété comme un signal satiétogène post-prandial.

\section{Existe-t-il une addiction aux lipides?}

Le choix de consommer un aliment plutôt qu'un autre dépend à la fois du plaisir qu'il procure et du bien-être ressenti après sa consommation. C'est ainsi qu'une souris préférera dans un premier temps une source d'AGLC non digestibles car estérifiés avec du sorbitol à une solution témoin non lipidique, puis cessera 


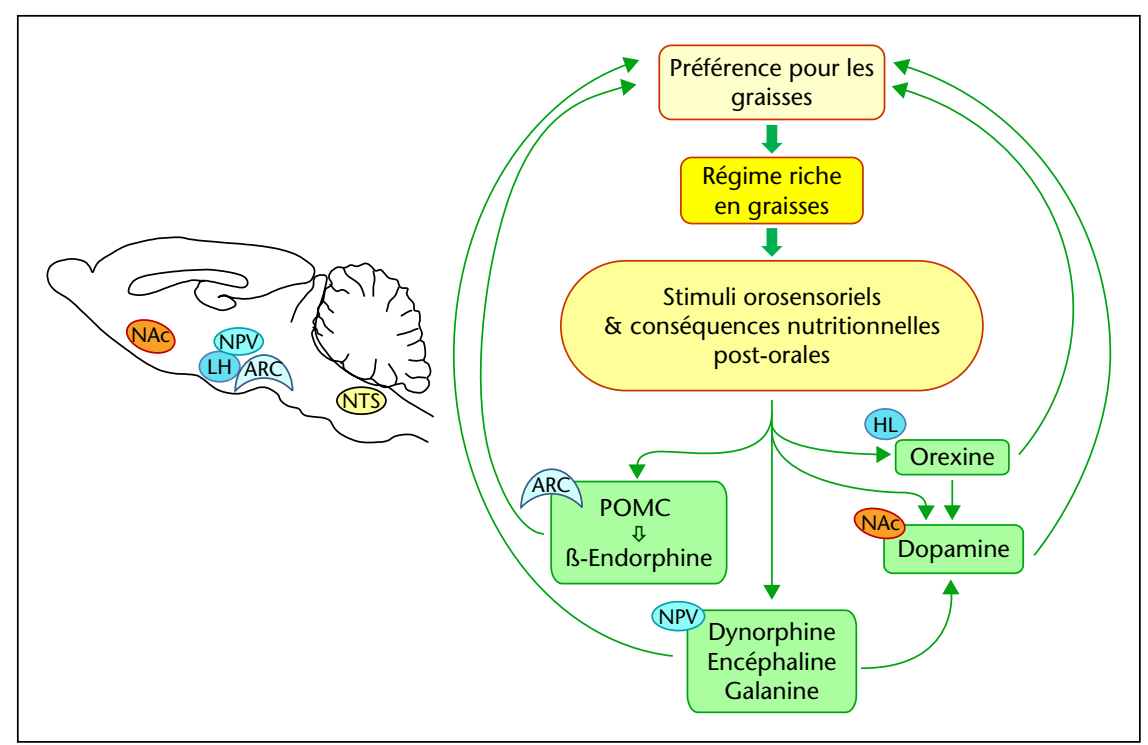

Figure 2. Origine de l'attraction des mammifères pour les lipides alimentaires.

Suite à la prise alimentaire de lipides, différentes zones du cerveau sont mises en jeu. Chez le rongeur, les lipides induisent la production de proopiomélanocortine (POMC) dans le noyau arqué de l'hypothalamus (ARC) ainsi qu'une augmentation de la concentration sérique et du fluide cerébrospinal en un dérivé de la POMC, la $\beta$-endorphine. La synthèse d'orexine est également induite dans I'hypothalamus latéral (HL). Les lipides induisent également l'expression paraventriculaire hypothalamique (NPV) de dynorphine et $\beta$-encéphaline. Ces molécules opiödes sont connues pour induire la prise alimentaire. Par ailleurs, il a été démontré que la mise en bouche de lipides, sans ingestion, induit la production de dopamine dans le noyau accumbens (NAc), zone impliquée dans les circuits de récompense.Cet ensemble complexe de voies de régulation contribue à l'attraction des rongeurs pour les graisses alimentaires.AGL, acides gras libres.

rapidement la consommation de ces lipides non nutritifs [20]. On peut donc penser que la préférence pour le « gras » peut conduire à une obésité quand les lipides consommés satisfont à la fois des besoins hédoniques et métaboliques. On constate en effet que des souris deviennent obèses quand on leur propose systématiquement de l'huile de maïs comme supplément optionnel à leur régime alimentaire habituel [21]. Cette préférence s'inscrivant dans la durée, on peut se demander s'il existe une addiction aux lipides chez le rongeur. Le fait que des souris consomment volontairement de l'huile au cours de tests de récompense (conditioning place preference, classiquement utilisés pour évaluer les comportements addictifs), en dépit d'un environnement hostile, supporte cette hypothèse [22] Ce comportement addictif n'est observé quand I'huile est délivrée directement dans l'estomac 60 minutes avant le conditionnement [22]. II semble donc que ce soit les informations d'origine orosensorielle qui jouent un rôle prépondérant dans ce comportement alimentaire de type addictif.

Les mécanismes moléculaires à l'origine de l'attraction pour les lipides commencent à être décryptés chez le rongeur. II implique à la fois le système opioidergique au niveau des noyaux arqué et paraventriculaire de l'hypothalamus et d'antagonistes sélectifs du récepteur $\mu$ supprime non seulement l'attirance pour le gras $[26,27]$ mais encore le conditionnement par I'huile de maïs lors des tests de comportement addictif [26]. Le récepteur $\mu$ des opiacés est donc impliqué dans l'attirance pour le gras. Le système dopaminergique semble également impliqué dans la régulation de la consommation de lipides chez le rongeur (figure 2). Des résultats déjà anciens indiquent que l'injection systémique d'antagonistes des récepteurs D1 et D2 de la dopamine diminue de façon dosedépendante la consommation d'une émulsion d'huile de maïs [28, 29]. Plus récemment, il a été montré qu'un repas fictif (car non ingéré) riche en huile de maïs provoque la production de dopamine par le noyau accumbens connu pour être impliqué dans le circuit de récompense [30]. Cette expérience, où les effets postingestifs sont exclus, démontre le rôle prépondérant de la détection orale des lipides dans l'activation d'une boucle de régulation positive renforçant la préférence pour les aliments riches en lipides. II est important de souligner que la sécrétion de dopamine par le noyau accumbens est également sous contrôle des peptides opiacés [31]. L'attraction pour les lipides chez le rongeur est donc la résultante d'un schéma régulateur complexe en cours d'élucidation impliquant de nombreux régulateurs et différentes zones du cerveau (figure 2).

dopaminergique au niveau de noyau accumbens (figure 2). Chez des rats conditionnées à prendre une émulsion d'huile de mais quotidiennement à heure régulière, une augmentation de l'expression de la proopiomélanocortine (POMC) dans le noyau arqué de I'hypothalamus et de l'orexine dans I'hypothalamus latéral est observée dans les trente minutes précédant la prise lipidique [23]. On constate également chez ces animaux une élévation de la concentration d'un opioïde dérivé de la POMC, la $\beta$-endorphine, dans le sérum et le fluide cérébrospinal, quinze minutes après cette prise d'huile [23]. Dans cette même espèce, un régime hyperlipidique provoque également une induction dans le noyau paraventiculaire de l'expression de la dynorphine et de la $\beta$-encéphaline, deux autres peptides opiacés régulateurs connus pour induire la consommation de lipides [24]. Ce résultat suggère l'existence d'une boucle de régulation positive. Ces opiacés stimulent la consommation de lipides qui, à leur tour, induisent la synthèse de ces peptides régulateurs. En accord avec cette hypothèse, l'injection centrale $d^{\prime}$ 'un agoniste du récepteur $\mu$ des opioïdes, le [D-Ala(2),NMe-Phe(4), Gly(5)-ol]-enkephalin (DAMGO), s'accompagne d'une augmentation de la consommation d'aliments riches en lipides chez le rat [25]. À l'inverse, I'administration

\section{Conclusion}

Certains mammifères, dont l'Homme, sont attirés par les aliments riches en graisses. Ce phénomène est la conséquence de la combinaison de la haute palatabilité des lipides associée à un « bien-être » métabolique (figure 3). La palatabilité des graisses est perçue au niveau oral via les sens de l'odorat, du toucher (perception de la texture) et de la gustation. Nos travaux démontrent de façon inédite que la protéine CD36 joue le rôle de lipido-récepteur gustatif chez la souris et le rat [8-10]. Une fois ingérés, les lipides sont aussi à l'origine d'une satisfaction métabolique en raison d'une absorption et $d^{\prime}$ un métabolisme très efficaces (production et stockage d'énergie). En revanche, les signaux neuroendocrines induits par la consommation de lipides ne semblent avoir qu'un faible pouvoir inhibiteur sur la prise alimentaire. Pris dans leur ensemble, ces facteurs régulateurs antagonistes favoriseraient plutôt la consommation d'aliments riches en lipides, ce qui pourrait constituer un avantage vital dans un environnement dominé par la précarité alimentaire (apport important d'énergie, d'acides gras indispensables et de vitamines liposolubles). En revanche, il pourrait favoriser l'apparition d'obésité dans un contexte de pléthore alimentaire permanente. 


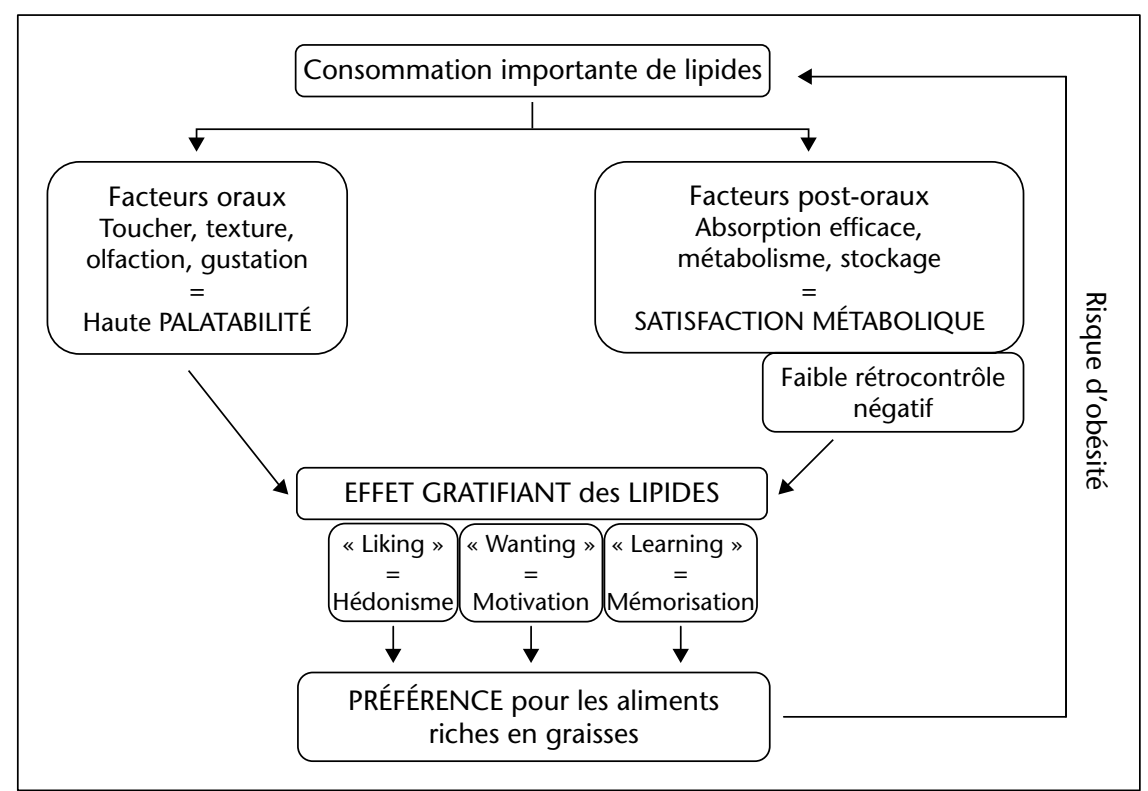

Figure 3. Facteurs influençant la préférence pour les lipides.

\section{RÉFÉRENCES}

1. NYSENBAUM AN, SMART JL. Sucking behaviour and milk intake of neonates in relation to milk fat content. Early Hum Develop 1982 ; 6 : 205-13.

2. HAMILTON CL. Rat's preference for high fat diets. / Comp Physiol Psychol 1964 ; 58 : 459-60.

3. TSURUTAM, KAWADAT, FUKUWATARIT, FUSHIKI T. The orosensory recognition of longchain fatty acids in rats. Physiol Behav $1999 ; 66$ : 285-8.

4. DREWNOWSKI A, BRUNZELL JD, SANDE K, IVERIUS PH, GREENWOOD MR. Sweet tooth reconsidered: taste responsiveness in human obesity. Physiol Behav 1985 ; 35 : 617-22.

5. MELA DJ, SACCHETTI DA. Sensory preferences for fats: relationships with diet and body composition. Am / Clin Nutr 1991 ; 53 : 908-15.

6. GAILLARD D, PASSILLY-DEGRACE P, LAUGERETTE F, BESNARD P. Sur la piste du "goût du gras" (On the track of the "taste of fat"). OCL 2006 ; 13 : 309-14.

7. GAILLARD D, PASSILLY-DEGRACE P, BESNARD P. Perception gustative des lipides alimentaires : paradigme et paradoxes (Gustatory perception of dietary lipids: paradigm and paradoxes). OCL $2008 ; 15: 41-5$.

8. LAUGERETTE F, PASSILLY-DEGRACE P, PATRIS B, ET AL. CD36 involvement in orosensory detection of dietary lipids, spontaneous fat preference, and digestive secretions. / Clin Invest $2005 ; 115$ : 3177-84.

9. GAILLARD D, LAUGERETTE F, DARCEL N, ET AL. The gustatory pathway is involved in CD36-mediated orosensory perception of long-chain fatty acids in the mouse. FASEB / $2008 ; 22$ : 1458-68.
10. EL-YASSIMI A, HICHAMI A, BESNARD P, KHAN NA. Linoleic acid induces calcium signaling, SRC Kinase phosphorylation, and neurotransmitter release in mouse CD36-positive gustatory cells. J Biol Chem 2008 ; 283 : 12949-59.

11. CHALE-RUSH A, BURGESS JR, MATTES RD. Evidence for human orosensory (taste?) sensitivity to free fatty acids. Chem Senses $2007 ; 32$ : 423-31.

12. RAYBOULD HE. Mechanisms of CCK signaling from gut to brain. Curr Op Pharmacol 2007 ; 7 : 570-4.

13. MALENDOWICZ LK, SPINAZZI R, MAJCHRZAK M, ET AL. Effects of prolonged cholecystokinin administration on rat pituitary-adrenocortical axis : role of the CCK receptor subtypes 1 and 2 . Int J Mol Med 2003 ; 12 : 903-9.

14. TSO P, LIU M. Ingested fat and satiety. Physiol Behav $2004 ; 81: 275-87$.

15. DOI T, LIU M, SEELEY RJ, WOODS SC, TSO P. Effect of leptin on intestinal apolipoprotein AIV in response to lipid feeding. Am / Physiol Regul Integr Comp Physiol 2001 ; 281 : R753-R759.

16. COLEMAN DL. Effects of parabiosis of obese with diabetes and normal mice. Diabetologia $1973 ; 9$ : 294-8.

17. MORTON G], CUMMINGS DE, BASKIN DG, BARSH GS, SCHWARTZ MW. Central nervous system control of food intake and body weight. Nature 2006 ; 443 : 289-95.

18. MIGRENNE S, MAGNAN C, CRUCIANIGUGLIELMACCI C. Fatty acid sensing and nervous control of energy homeostasis. Diabetes Metab 2007 ; 33 : 177-82.

19. WANG R, CRUCIANI-GUGLIELMACCI C, MIGRENNE S, MAGNAN C, COTERO VE, ROUTH VH. Effects of oleic acid on distinct populations of neurons in the hypothalamic arcuate nucleus are dependent on extracellular glucose levels. I Neurophysiol 2006; 95 : 1491-8.

20. SUZUKI A, YAMANE T, IMAIZUMI M, FUSHI$\mathrm{KI}$ T. Integration of orosensory and postingestive stimuli for the control of excessive fat intake in mice. Nutrition $2003 ; 19: 36-40$.

21. TAKEDA M, IMAIZUMI M, SAWANO S, MANA$B E Y$, FUSHIKI T. Long-term optional ingestion of corn oil induces excessive caloric intake and obesity in mice. Nutrition 2001 ; 17 : 117-20.

22. IMAIZUMI M, TAKEDA M, FUSHIKI T. Effects of oil intake in the conditioned place preference test in mice. Brain Res $2000 ; 870$ : 150-6.

23. MIZUSHIGE T, KAWAIT, MATSUMURA S, ET AL. POMC and orexin mRNA expressions induced by anticipation of a corn-oil emulsion feeding are maintained at the high levels until oil ingestion. Biomed Res (Tokyo, Jpn) 2006 ; 27 : 227-32.

24. CHANG GQ, KARATAYEV O, AHSAN R, GAYSINSKAYA V, MARWIL Z, LEIBOWITZ SF. Dietary fat stimulates endogenous enkephalin and dynorphin in the paraventricular nucleus : role of circulating triglycerides. Am J Physiol Endocrinol Metab 2007 ; 292 : E561-E570.

25. WILL MJ, FRANZBLAU EB, KELLEY AE. Nucleus accumbens mu-opioids regulate intake of a high-fat diet via activation of a distributed brain network. J Neurosci 2003 ; 23 : 2882-8.

26. IMAIZUMI M, TAKEDA M, SAWANO S, FUSHI$\mathrm{KI}$ T. Opioidergic contribution to conditioned place preference induced by corn oil in mice. Behav Brain Res 2001 ; 121 : 129-36.

27. MIZUSHIGE T, MATSUMURA S, YONEDA T, TSUZUKI S, INOUE K, FUSHIKI T. Daily increase of fat ingestion mediated via mu-opioid receptor signaling pathway. Biomed Res (Tokyo, Jpn) 2006 ; 27 : 259-63.

28. WEATHERFORD SC, SMITH GP, MELVILLE LD. D-1 and D-2 receptor antagonists decrease corn oil sham feeding in rats. Physiol Behav $1998 ; 44: 569-72$.

29. WEATHERFORD SC, GREENBERG D, GIBBS I, SMITH GP. The potency of D-1 and D-2 receptor antagonists is inversely related to the reward value of sham-fed corn oil and sucrose in rats. Pharmacol Biochem Behav 1990; 37 : 317-23.

30. LIANG NC, HAJNAL A, NORGREN R. Sham feeding corn oil increases accumbens dopamine in the rat. Am J Physiol Regul Integr Comp Physiol 2006 ; 291 : R1236-R1239.

31. SPANAGEL R, HERZ A, SHIPPENBERG TS. The effects of opioid peptides on dopamine release in the nucleus accumbens : an in vivo microdialysis study. J Neurochem 1990 ; 55 : 1734-40. 